\title{
EVALUACIÓN PROSPECTIVA DE LA EFICIENCIA ECONÓMICA DE LA PRODUCCIÓN DE FRIJOL EN LA PROVINCIA SANTIAGO DE CUBA.
}

\author{
Lic. Ulises Pacheco Feria. \\ Profesor titular y Dr.C en Ciencias Económicas por la Universidad de Oriente. \\ Máster en Economía por la Universidad de Carletón, Canadá. \\ Fac. Ciencias Económicas y Empresariales de la Universidad de Oriente. Santiago de Cuba, Cuba. \\ upacheco@uo.edu.cu \\ Lic. Rosa Marina Castellanos Dorado. \\ Profesora auxiliar. Máster en Economía por la Universidad de Valencia, España. \\ Dra. C en Ciencias Económicas por la Universidad de Oriente. \\ Fac. de Ciencias Económicas y Empresariales de la Universidad de Oriente Santiago de Cuba, Cuba. \\ rosy@uo.edu.cu

\section{Lic. Milagros Morales Pérez.} \\ Profesora Titular. Máster en Economía por la Universidad de Valencia, España. Dra. C en Ciencias \\ Económicas por la Universidad de Oriente. Institución de adscripción: Facultad de Ciencias \\ Económicas y Empresariales de la Universidad de Oriente. Santiago de Cuba, Cuba. \\ milagros@uo.edu.cu
}

\section{RESUMEN}

El objetivo del artículo es evaluarla eficiencia económica de la producción de frijol, con un enfoque prospectivo en la provincia Santiago de Cuba. La evaluación se realizó mediante el Método de impactos cruzados y el Método multicriterio y política de Michel Godet (1994) y permitió diseñar siete escenarios (pesimista, optimista, apuesta y cuatro alternativos). Se demostró que en el escenario apuesta se cumplen cinco de las seis hipótesis por lo que se evalúan de bien la eficiencia productiva y la asignativa, y de regular la distributiva. Las políticas diseñadas contribuyen al logro del objetivo propuesto por el Programa de Desarrollo Integral de Granos en la provincia Santiago de Cuba, al incremento de los rendimientos y la producción de granos con una reducción significativa de los costos, logrando producciones sostenibles en rubros que constituyen sustitución de importaciones (maíz y frijol) para, finalmente, coadyuvar al incremento del consumo.

PALABRAS CLAVE: Eficiencia económica, eficiencia productiva, eficiencia asignativa, eficiencia distributiva, prospectiva, escenario apuesta. 


\title{
PROSPECTIVE EVALUATION OF THE ECONOMIC EFFICIENCY OF BEAN PRODUCTION IN THE PROVINCE OF SANTIAGO DE CUBA.
}

\begin{abstract}
The objective of the article is to evaluate the economic efficiency of the bean production, with a prospective focus in Santiago de Cuba. The evaluation was carried out by means of the Method of crossed impacts and the Method multi-criterion and politics of Michel Godet (1994) and it allowed to design seven scenarios (pessimist, optimist, bet and four alternative). It was demonstrated the best scenario performs five of the six hypotheses therefore the productive and allocative efficiency gets a good evaluation and the distributive a regular evaluation. The designed policies contribute to the achievement of the objective proposed by the Program of Integral Development of Grains in Santiago de Cuba, to the increment of the yields and the production of grains with a significant reduction of the costs, achieving sustainable productions in items that constitute substitution of imports (corn and bean) in order to cooperate to the increment of the consumption.
\end{abstract}

KEYWORDS: Economic efficiency, productive efficiency, allocative efficiency, distributive, prospective efficiency, bet scenario.

\section{INTRODUCCIÓN}

El frijol común, Phaseolus vulgaris L., es, dentro de las leguminosas de grano, una de las especies más importantes para el consumo humano. La Organización de las Naciones Unidas para la Alimentación y la Agricultura (FAO) declara que es considerado una de las principales fuentes de proteínas, especialmente para aquellas poblaciones de bajos recursos, y dentro de los productos básicos de la seguridad alimentaria de las áreas rurales y de bajos ingresos.

En Cuba, el frijol junto con el arroz y las viandas, constituye un alimento de preferencia en la dieta cotidiana; y es, por demás, uno de los elementos proteicos básicos que la conforman. Por tal motivo, es importante asegurar el cumplimiento de los programas de producción de frijol en el país. Los programas, por su parte, deben reflejar adecuadamente la necesidad de perfeccionar las políticas y los mecanismos que impulsen un incremento de la eficiencia de la producción de frijol en los territorios, en correspondencia con las características propias de cada región; a saber: a) la dotación de factores productivos (tierra, fuerza de trabajo y parque tecnológico disponible); b) situación alimentaria territorial; y c) los costos tangibles y de oportunidad que entraña el desarrollo de la producción de este grano en cada localidad. 
Santiago de Cuba es una de las provincias en Cuba que requiere especial atención para el cumplimiento del programa de granos considerando que muestra uno de los volúmenes más bajos de producción y rendimientos del cultivo de esta leguminosa.

Un análisis de los principales indicadores de la eficiencia económica revela que el rendimiento promedio anual para el territorio es de apenas 0,52 toneladas por hectárea, muy por debajo de la media nacional; los costos de producción son altos; los costos de oportunidad de la producción de frijol con respecto al maíz son elevados; la remuneración efectiva no cubre suficientemente las necesidades básicas de un trabajador agrícola y su familia; el consumo es insuficiente; existe insatisfacción de los consumidores en cuanto a la cantidad, calidad, precio y preferencias con el producto.

Por tal razón, la producción de frijol junto al maíz, constituyen los objetivos más importantes del Programa de Desarrollo Integral de Granos (PDIG) de la provincia hasta el 2030. El PDIG, tiene como objetivo incrementar los rendimientos y la producción de granos con el mínimo de costos, aplicando una agricultura sustentable y sostenible. Sin embargo, el mismo encuentra un conjunto de obstáculos para la satisfacción de la demanda interna, entre los que se destacan: la prevalencia de condiciones de sequía agrícola y de altas temperaturas; más del $50 \%$ de los suelos están clasificados, desde el punto de vista agroproductivo, como muy poco fértiles; el territorio no cuenta con una tradición frijolera comparable con otras zonas del país y la eficiencia económica es baja.

A partir de la situación actual descrita de la producción de frijol en la provincia resulta necesario e importante diseñar políticas que permitan alcanzar el escenario apuesta en el largo plazo (2030), lo que constituye una ayuda a los gestores de políticas en la toma de decisiones.

El enfoque prospectivo para la evaluación de la eficiencia económica de la producción de frijol en la provincia, supone una adecuación al enfoque tradicional de la prospectiva, según la literatura especializada, pues es aplicada, en este caso, a un programa mesoeconómico, el PIDG, en correspondencia con las características del territorio y no al desarrollo de un territorio o región como un ejercicio de planeación integral, además evalúa la eficiencia económica, entendida esta última como una integración de sus tres tipologías esenciales (productiva, asignativa y distributiva).

Se hace énfasis en las tipologías esenciales de eficiencia económica porque en la literatura especializada existen diferentes tipos de eficiencia, entre los que se destacan: la eficiencia productiva o técnica (Koopmans, 1951), (Debreu, 1951), (Farrell, 1957), (Forsund y Hjalmarsson, 1974), (Lerner, 1951), (Cachanosky, 2012).La asignativa(Farrell, 1957), (Forsund y Hjalmarsson,1974), (Lerner, 1951), (Sellers, 2005), (García, 2015).La eficiencia dinámica(Cachanosky, 2012). La eficiencia coordinativa e informativa (Lindbeck, 1977). La eficiencia de escala ${ }^{1}$ y la eficiencia distributiva (Lerner, 1951 y Leibestein, 1966).

De todos los tipos de eficiencia mencionados los más reconocidos son la eficiencia productiva o técnica y la asignativa. Independientemente de la amplia tipología que sobre la eficiencia económica existe, a juicio de los autores, los tipos que esencialmente explican este concepto son: la productiva, la asignativa y la distributiva, por constituir la

\footnotetext{
${ }^{1}$ Färe, Grosskopf y Lovell consideran que la eficiencia técnica puede ser de escala (1994), otros como Forsund y Hjalmarsson (1974) clasifican la eficiencia de escala como un tipo específico de eficiencia.
} 
producción y la distribución fases de la base económica que anteceden al consumo. La eficiencia dinámica, coordinativa e informativa y la eficiencia de escala son derivaciones de la eficiencia productiva y de la técnica.

La necesidad de un análisis integrado de las tres tipologías de eficiencia antes mencionadas, tiene como fundamento teórico la concepción de Carlos Marx sobre la producción material, como proceso que, en su definición más amplia, integra todas las fases de la reproducción de la riqueza material; a saber: la producción, la distribución, el cambio y el consumo.

Existe una relación sistémica entre estos tres tipos de eficiencia. La eficiencia productiva condiciona la asignativa y la distributiva, pues no pueden asignarse y distribuirse recursos que no sean producidos y, a su vez, una asignación y distribución eficiente de los factores y de los resultados de la producción, garantiza obtener un nivel de output con la menor cantidad de insumos posibles.

Por tal razón, la evaluación prospectiva de la eficiencia económica de la producción de frijol centrará la atención en las tres tipologías esenciales que asume la investigación. Y es definida como la integración (sistémica), de sus tipologías esenciales: la eficiencia productiva, la asignativa y la distributiva. Significa, que la eficiencia económica es una condición en que se obtiene un nivel de output con la menor cantidad de insumos posibles, por una asignación óptima de factores, y la remuneración se realiza con arreglo al aporte, ordenándose los mercados para facilitar el consumo.

Nótese, que el concepto integra los tres tipos de eficiencia que, como se mencionó, la investigación asume como esenciales para definir el fenómeno. Debe de considerarse además los factores determinantes por tipología, es decir:

- La eficiencia técnico-productiva en la que es necesario obtener un nivel de output con la menor cantidad de insumos posibles y por tal razón los factores determinantes a considerar son: la producción, el costo, el rendimiento y la productividad.

- La eficiencia asignativa prevé realizar elecciones en cuanto a una combinación optima de factores y por tal motivo los factores determinantes son: dotación de factores y costo de oportunidad

- La eficiencia distributiva debe lograr alinear la remuneración, aporte laboral y mercado en función del consumo, por tanto los factores determinantes a tener en cuenta son: relación remuneración efectiva e ingreso neto, incentivos y relación entre demanda efectiva y consumo.

La incorporación de la evaluación prospectiva en el estudio de la eficiencia de la producción agrícola, con el enfoque antes expuesto, es importante si se considera el alto nivel de incertidumbre que gravita sobre este sector, y en particular sobre la producción de frijol, porque este es un cultivo muy vulnerable a las condiciones naturales y a factores no controlables por el hombre. La evaluación prospectiva contribuye a reducir la incertidumbre y a adoptar decisiones en aras de una aproximación al escenario deseado, como una apuesta en la construcción participativa del futuro.

A pesar de los bajos rendimientos de la producción de frijol en el ámbito nacional e internacional, de las propiedades nutricionales de este grano y de la importancia de incorporar la prospectiva a estudios de esta naturaleza, son limitadas las investigaciones con este enfoque. Y así lo corroboró la bibliografía consultada. 
Se revisaron 16 estudios, siete internacionales (España, Chile, Venezuela, empresas de América Latina y el Caribe) y nueve nacionales (Villa Clara, Cienfuegos, Matanzas, Nicaro y Granma), que son: "Análisis de la eficiencia técnica y asignativa a través de las fronteras de costes: una aplicación a los hospitales del INSALUD”, España, (García, 2002); "La eficiencia en la asignación de recursos destinados a la Educación Superior. El caso de la Universidad La Laguna", España (Martín, 2006); "Productividad y eficiencia en la distribución minorista en España", (Sellers, 2005); "Tres ensayos sobre eficiencia económica y crecimiento regional: Capacidad empresarial, externalidades y estructura productiva”, España, (Gómez, 2014); "Eficiencia y su medición en prestadores de servicios de agua potable y alcantarillado", CEPAL, (Ferro y Romero, 2011); "Estudio comparativo de la eficiencia técnica de sistemas ganaderos de doble propósito en las zonas agroeconómicas de los municipios zulianos de la cuenca del Lago de Maracaibo, Venezuela”, (Urdaneta, Dios y Cañas, 2013); “Análisis de la eficiencia en Chile”, (Planck, 2011); "Procedimiento para la evaluación de la eficiencia técnica en la transportación de caña en las UBPC cañeras de la provincia Villa Clara", Cuba, (Romeu y Rodríguez, 2008); "Eficiencia técnica en unidades lecheras", Cuba, (Herrera y Barrios, 2013). "Metodología para el análisis de la productividad en la producción de arroz", Cuba (Guerra, 2011); "Productividad y eficiencia en la economía cubana: una aproximación empírica", (Doimeadiós y Sánchez, 2015);“Evaluación de la eficiencia técnica de la producción de viandas en las empresas agropecuarias Benito Juárez y Osvaldo Herrera", (Cancio y Barrios C, 2010); "Niveles de eficiencia de las policlínicas de Matanzas según ADE", Cuba (García, Sánchez, Chadiano y Núñez, 2007);“La medición de la eficiencia productiva en policlínicos cubanos: un caso de estudio", (García y Sarrias, 2008); “Midiendo la eficiencia en la actividad turística mediante el análisis envolvente de datos, Cuba, (Montes de Oca, 2009); "Indicadores para el análisis de la eficiencia económica de empresas productoras de níquel. Caso de estudio empresa "Cdte. René Ramos Latour" de Nicaro", Cuba, (Bell y Sarmiento, 2011).

La revisión centró la atención en los siguientes aspectos: sector en que se realiza el estudio, tipo de eficiencia que se estudia; factores que se evalúan por tipologías de eficiencia y el método utilizado.El análisis crítico realizado, atendiendo a los momentos mencionados anteriormente permite resumir las siguientes ideas:

- En cuanto a los sectores, debe destacarse que la mayoría de los estudios se realizan en el sector de los servicios: en hospitales del INSALUD, Valladolid, España; en 190 hospitales públicos de Chile, más la región Metropolitana de ese país; en policlínicos cubanos (Matanzas y Cienfuegos); en la Educación Superior (el caso de la Universidad La Laguna, en Tenerife, España); en la distribución minorista en regiones españolas; en prestadores de servicios de agua potable y alcantarillado por la Comisión Económica para América Latina y el Caribe (Cepal), en empresas de la región; en hoteles y restaurantes cubanos. También se han realizado estudios de eficiencia en el sector productivo: empresas de Barcelona, España; sistemas ganaderos de los municipios zulianos de la cuenca del Lago de Maracaibo, Venezuela; en las UBPC cañeras de la provincia Villa Clara; en un grupo de vaquerías de la Empresa Pecuaria Genética "Valle del Perú", en Villa Clara; en la producción de arroz en la provincia 
Granma; en la industria manufacturera cubana, en la producción de viandas de empresas agropecuarias en Villa Clara y en empresas productoras de níquel, Nicaro.

- Dentro del sector productivo y agrícola se estudia la eficiencia de la producción de arroz, de caña y de viandas. A pesar de las bondades que tiene el frijol como grano, y de que los rendimientos nacionales e internacionales son bajos, no se encontró ningún caso de estudio al respecto, lo que evidencia un vacío en la investigación realizada sobre el tema.

- Los tipos de eficiencia analizados fueron: los 16 estudios analizan la eficiencia técnica o productiva, tres miden la eficiencia asignativa; dos, la eficiencia global o total; y dos, la eficiencia de escala. Solo tres estudian conjuntamente la productiva y la asignativa (Valladolid y la Universidad La Laguna, en España) y el estudio de la Cepal. Este último también analiza la eficiencia total, al igual que se realiza en el grupo de vaquerías de Villa Clara. La eficiencia de escala se estudió en los 190 hospitales públicos en Chile y en las unidades lecheras de Villa Clara.

Este enfoque integral debe fundamentar el proceso de evaluación. Sin embargo, ningún estudio, de los revisados, evalúa los tres tipos de eficiencia que esta investigación asume como esenciales para un enfoque integral. Las limitaciones señaladas han sido, fundamentalmente, a aquellos estudios en los que el objetivo declara el análisis de la eficiencia económica y solo se evalúa una u otra tipología.

- Referido a los factores que utilizan los diferentes estudios para analizar cada tipo de eficiencia, debe destacarse que en el caso de la eficiencia productiva se proponen indicadores relacionados con la especificidad de cada proceso productivo. En el caso de las factores para medir la eficiencia asignativa, en la aplicación a los hospitales del INSALUD se constata hasta qué punto las proporciones en que se emplean los factores se alejan de los óptimos. En las otras dos investigaciones en que se declara el análisis de este tipo de eficiencia: el estudio de la Cepal y la eficiencia en la asignación de recursos destinados a la Educación Superior, no se precisa qué factores fueron empleados para tales fines.

- Relacionado con el método, en la revisión realizada, en el área internacional, de los siete casos consultados emplearon seis el método Análisis Envolvente de Datos, y de los estudios nacionales, de nueve, lo utilizaron siete. Solo en el caso de la "Eficiencia y su medición en prestadores de servicios de agua potable y alcantarillado", Cepal se realizó la medición y la evaluación de la eficiencia mediante la comparación de desempeño. En ningún caso se empleó la prospectiva como método.

Por tanto, no se encontró, en la literatura especializada, ningún estudio acerca de la eficiencia económica de la producción de frijol que incluya la prospectiva ${ }^{2}$ y que

\footnotetext{
${ }^{2}$ La prospectiva ha sido ampliamente utilizada en diferentes investigaciones. Si se desea profundizar revisar: De Somoza, A. 2015. "Herramientas para la formulación de políticas: el Análisis Prospectivo en la Construcción de Escenarios Energéticos y el uso de modelos para su formulación", http://www.nodo50.org. De Sarrías P. 2008. "Aplicación del enfoque prospectivo para la gestión del desarrollo local: caso de estudio". En Revista DELOS, Vol. 1, No. 2. http:// www.eumed.net. De Medina, V. 2002. "Bases desde la Prospectiva-Previsión Territorial para el diseño del Plan Maestro del Desarrollo Integral y Sostenible del Valle del Cauca. Universidad del Valle. Quito. Ecuador". http://www.es.slideshare.net
} 
considere, además, las tres tipologías esenciales de la eficiencia económica mencionadas. Lo anterior evidencia la existencia de un vacío en la bibliografía y justifica que el objetivo del artículo sea evaluar la eficiencia económica de la producción de frijol, con un enfoque prospectivo, en la provincia Santiago de Cuba.

La prospectiva no se compromete en predecir el futuro, sino que se extiende en la exposición (narrativa) de los posibles derroteros que tenga el desarrollo de un evento o proceso, en condiciones de riesgo e incertidumbre. Es una herramienta analítica para adoptar decisiones más acertadas en situaciones de cambios muy dinámicos y de compleja interacción de múltiples variables, mitigando la incertidumbre y atenuando la posibilidad de errores de planificación.

La construcción de escenarios no es una predicción infalible e incuestionable de lo que va a suceder en el futuro, sino una aproximación a lo que podría suceder y, por ende, es un instrumento para decidir mejor en un entorno cambiante, disminuyendo así el margen de error. Por ello, una de las cuestiones más relevantes que no puede perderse de vista en la construcción del escenario es la clara diferenciación de los escenarios más probables y de los más deseables. Solo así podrán trazarse políticas que sean coherentes, acercando las líneas de deseo a las posibilidades reales que las condiciones objetivas permiten alcanzar.

El marco estratégico de la producción de frijol en la provincia Santiago de Cuba se desarrolla en condiciones en las que constituye una prioridad la seguridad alimentaria de la población nacional y local. Al mismo tiempo, se desenvuelve a tono con los Lineamientos de la Política Económica y Social del Partido Comunista de Cuba para actualizar el modelo económico cubano, siendo su expresión concreta el PDIG de la provincia Santiago de Cuba hasta el año 2030, el cual incluye, como una de las líneas priorizadas, la producción de frijol.

El programa abarca dos polos productivos: el polo productivo de granos de Contramaestre (que incluye las producciones de los municipios Palma Soriano y Contramaestre) y el polo productivo de Granos de Songo-La Maya (que incluye las producciones de La Maya, Los Reynaldo, San Luis, Segundo Frente y Santiago de Cuba). A partir del diagnóstico económico del programa que evalúa el comportamiento de la producción, áreas cosechadas y los rendimientos de frijol y de maíz, por empresas y formas productivas en el año base utilizado (2014), fueron seleccionadas por el Ministerio de la Agricultura 11 empresas de la agricultura con posibilidades de aportar grandes volúmenes de producciones por sus características y tradiciones en el cultivo de los granos. Del polo productivo de granos de La Maya se eligieron cinco empresas, y del polo productivo de granos de Contramaestre, seis.

EI PDIG tiene como objetivo incrementar los rendimientos y la producción con el mínimo de los costos aplicando una agricultura sustentable para contribuir al Programa Nacional de Producción de Granos, considerando todos los eslabones de la cadena, desde la 
logística, la siembra, la tecnología del cultivo, la cosecha y el beneficio hasta la comercialización con productos de calidad.

La evaluación prospectiva de la eficiencia económica de la producción de frijol en el territorio concibe el diseño de los escenarios futuros como su herramienta metodológica fundamental. Los escenarios construidos deben ser coherentes, por lo que la narrativa tiene que estar articulada de manera lógica y no deben salirse del propósito esencial que los define. Además, como se ha mencionado, la eficiencia económica será evaluada como resultado de la dinámica hipotética de los factores determinantes de cada tipología de eficiencia.

\section{METOdología}

Entre los métodos más empleados para la construcción de escenarios está el método de impactos cruzados (SMIC), y para el diseño y selección de políticas el Método Multicriterio y Política (MULTIPOL), ambos métodos de Michel Godet (1994). Tomando como referencia los métodos mencionados la evaluación prospectiva de la eficiencia económica de la producción de frijol se realizará mediante los pasos siguientes:

Paso 1: Selección del grupo de expertos.

Para la selección del grupo de expertos se utilizó el conocido método, Coeficiente de Competencia. Dicho método comprende inicialmente la evaluación del Coeficiente de Conocimiento $\left(\mathrm{K}_{\mathrm{c}}\right)$ sobre el tema en cuestión, y se considerará el rango seleccionado por el experto en una escala que oscila entre uno (no conocer ni tener información sobre el tema) y diez (tener elevado dominio del tema). De esa manera se tabulan los expertos por las filas y la escala por las columnas. El resultado obtenido se procesa a través de la fórmula:

$K_{c}=n(0.1)$

Donde $\left(K_{c}\right)$ indica el Coeficiente de conocimiento o Información y $(n)$ es el número escogido por el experto en la escala antes comentada para autoevaluarse.

Una vez calculado el Coeficiente de Conocimiento se procede a valorar el nivel de argumentación o fundamentación que tiene el experto sobre el tema que se va a estudiar, y se calcula el Coeficiente de Argumentación de cada experto $\left(\mathrm{K}_{\mathrm{A}}\right)$ :

$K_{A}=\sum n_{i}=\left(n_{1}+n_{2}+n_{3}+n_{4}+n_{5}\right)$

Donde (ni) es el valor asignado por el experto, sobre la base de una escala de alto, medio o bajo, a cada fuente de argumentación (i) propuesta por el investigador ${ }^{3}$.En este caso las fuentes de argumentación a evaluar son: estudios teóricos realizados sobre el tema en

\footnotetext{
${ }^{3} \mathrm{~A}$ los efectos de esta metodología solo se consideran cinco fuentes de argumentación, aunque es válido aclarar que pueden emplearse tantas fuentes como el investigador estime conveniente, lo cual puede poseer un doble efecto: flexibilizar el espectro de opinión del experto y complejizar el cálculo del coeficiente. De igual forma, la escala propuesta en esta metodología para ponderar la importancia de las fuentes, es susceptible de modificación.
} 
cuestión; experiencia de trabajo; trabajos de investigación consultados relacionados con el tema; Comisiones de trabajo en las que ha participado e intuición del individuo.

Una vez calculado el Coeficiente de Conocimiento $(K c)$ y el Coeficiente de Argumentación $\left(K_{A}\right)$, se procede a calcular el Coeficiente de Competencia $(K)$, que determina qué experto será incluido en el estudio y cuál será excluido (anexo 4).

$K=0.5\left(K_{c}+K_{a}\right)$

En correspondencia:

Si $0,8<K<1,0$ el Coeficiente de Competencia del experto es Alto. Si $0,5<K<0,8$ el Coeficiente de Competencia del experto es Medio. Si $\mathrm{K}<0,5$ el Coeficiente de Competencia del experto es Bajo.

Es recomendable escoger aquellos expertos cuyos Coeficientes de Competencia sean altos, o al menos, medios. Un último paso ilustrativo de la calidad científica de los expertos seleccionados puede ser el cálculo del promedio ponderado de los coeficientes individuales de competencia de los expertos seleccionados:

$K=\frac{\sum K_{i}}{n}$

Donde $K_{i}$ representa el Coeficiente Individual y $n$ el número de expertos.

Una vez seleccionado los expertos que trabajaran en el estudio, se procede a formular las hipótesis, segundo paso de la metodología.

Paso 2: Formulación de las hipótesis.

Para la formulación de las hipótesis debe tenerse en cuenta que estas:

- Representan los factores determinantes del fenómeno estudiado.

- Expresan el comportamiento del sistema en la actualidad

- Deben ser formuladas considerando el futuro.

Por tal razón, este paso "implica transformar los factores determinantes en hipótesis" (Cely B., A., 1999: 33), combinando la situación actual y el futuro.

El software SMIC combina las hipótesis simples tomando una restricción binaria (se cumple o no se cumple la hipótesis), al tiempo que admite sólo seis hipótesis iniciales; por consiguiente, la cantidad máxima de escenarios se define entonces como $2^{6}=64$.

Los expertos deben recibir la información necesaria para formular las hipótesis, en términos del comportamiento de los factores determinantes en la actualidad y su proyección futura. La formulación de las hipótesis conduce a la asignación de las probabilidades de ocurrencia, siguiente paso.

Paso 3: Asignar las probabilidades (simples y condicionales) de ocurrencia de las hipótesis. 
La asignación de probabilidades simples de ocurrencia de las hipótesis se realiza tomando en cuenta la definición clásica de probabilidades de Laplace según la cual, la probabilidad del evento A:

$$
P(A)=\frac{\text { No de resultados favorables al evento } A}{\text { Número de resultados posibles }}
$$

La asignación de probabilidades asumirá la escala siguiente (Cely, 1999):

$\begin{array}{ll}\text { Evento muy probable } & 0,9 \\ \text { Evento probable } & 0,7 \\ \text { Evento de dudosa ocurrencia } & 0,5 \\ \text { Evento improbable } & 0,3 \\ \text { Evento muy improbable } & 0,1\end{array}$

La asignación de probabilidades condicionales a la ocurrencia de las hipótesis se realiza con arreglo a la escala anterior. Se asume la existencia de dependencia entre los eventos (hipótesis) y se calculan las probabilidades condicionales del cumplimiento de una hipótesis ( $\mathrm{H} 1)$ dado el cumplimiento de otra $(\mathrm{H} 2)(P(H 1 / H 2))$.Luego se realiza el mismo cálculo para el evento complementario, es decir la probabilidad de que se cumpla una hipótesis ( $\mathrm{H} 1)$ dado el no cumplimiento de la otra $(\mathrm{H} 2)\left(P\left(H 1 / H 2^{-1}\right)\right){ }^{4}$. El software SMIC permite corregir las probabilidades asignadas por los expertos (llamadas probabilidades brutas), convirtiéndolas en coherentes (probabilidades corregidas); de esta manera el SMIC ajusta las probabilidades asignadas, a los principios clásicos que deben cumplir las probabilidades, desde el punto de vista matemático. Para obtener este resultado el SMIC utiliza la minimización cuadrática de las diferencias entre probabilidades brutas y corregidas, bajo restricciones lineales.

A partir de las probabilidades corregidas el software Prob-expeJt realiza un análisis de sensibilidad para mostrar las relaciones de interdependencia entre las hipótesis. El paso que sigue centra su análisis en ese análisis.

Paso 4: Análisis de sensibilidad.

EI SMIC realiza un análisis de sensibilidad a partir de los coeficientes de elasticidad internamente definidos, determinando la magnitud y sentido de las variaciones de una hipótesis determinada ante la variación en un $10 \%$ de la probabilidad de ocurrencia del resto. Adicionalmente, permite conocer cuáles son las hipótesis más influyentes dentro del sistema, y cuáles las más dependientes.

\footnotetext{
${ }^{4} \mathrm{Si}$ se desea profundizar en este tema, revisar de Cely, A. V. (1999). "Metodología de los escenarios para estudios prospectivos". En Revista Ingeniería e Investigación, No. 44. Recuperado de http://www.bdigital.unal.edu.co
} 
Este software identifica la cantidad de escenarios que suman acumuladamente el $80 \%$ de las probabilidades; sin embargo, los expertos pueden seguir una conducta independiente, determinando que cantidad de escenarios tomar para su análisis y evaluación. (Godet, 2000; y Godet, Durance, 2011) ${ }^{5}$.

Los expertos deben escoger dentro del total de escenarios, el subgrupo que acumula el $50 \%$ de las probabilidades que estará definido como núcleo tendencial para su análisis; el resto será definido como escenarios alternativos. Del subgrupo tendencial se identifica el escenario deseado.

La metodología SMIC-EXPERT considera que, en lo fundamental, se selecciona una cantidad de escenarios tales que en su conjunto representen el $80 \%$ de las probabilidades de ocurrencia; sin embargo, este porciento puede variar y, por tanto, la cantidad de escenarios evaluados, de acuerdo al análisis que se haga a posteriori por parte de los expertos (Godet, 2000).

Paso 5: Diseño y selección de política para mejorar la eficiencia económica de la producción de frijol.

El diseño de políticas debe encaminarse a orientar la toma de decisiones en el presente para alcanzar el escenario apuesta en mediano y largo plazo. Desde este punto de vista, permite mitigar la incertidumbre y orientar más eficazmente a los decisores de política vinculados a la eficiencia de la producción de frijol.

Las políticas no pretenden alcanzar un escenario utópico sino un "segundo mejor", el futurible (el mejor de todos los futuros posibles), que se encuentra entre este y el escenario pesimista, lo que evidencia el pragmatismo que acompaña a cualquier diseño de política que se precie de realizable. En este sentido, la política sigue siendo como acuñó el pensamiento clásico: "el arte de lo posible", en contraposición a la realidad soñada o idílica que resulta, en un contexto concreto, un escenario inalcanzable.

Para el diseño y elección de políticas se utilizó, como se mencionó, el Método Multicriterio y Política (MULTIPOL). El método tiene como objetivo elegir para cada escenario cuáles serían las políticas más importantes, y dentro de ellas, las acciones. Compara diferentes acciones o soluciones a un problema a partir de criterios y de políticas múltiples, para de esta forma apoyar a la toma de decisiones. Cada acción es evaluada en función de los criterios por medio de una escala, en un rango de cero al 20. Esta evaluación puede realizarse a través de cuestionarios o consultando a los expertos, con la búsqueda de consenso. Luego se evalúan las políticas y escenarios en función de los criterios. En este

\footnotetext{
${ }^{5}$ Un análisis detallado acerca de la matriz de sensibilidad se puede consultar en la obra de Michel Godet, (1994). De la Anticipación a la Acción. Manual de Prospectiva Estratégica, en el Capítulo 5, epígrafe 5.2, inciso C, pág. 158 Barcelona: España, Editorial Mar Combo y de Godet, M y Durance, P. 2011. La prospectiva estratégica para las empresas y los territorios. https://es.scribd.com
} 
caso, como se trata de un juego de peso de criterios la suma en línea debe ser siempre la misma, igual a 100. A partir de esta información se obtiene: la evaluación del resultado de las acciones en función de las políticas y la evaluación de las políticas en función de los escenarios. Resumiendo, el método tiene tres momentos:

- Definir, por parte del grupo de expertos, las políticas, acciones y criterios.

- Evaluar las políticas, acciones y escenarios con respecto a cada criterio, por parte de los expertos individualmente, y consolidar las respuestas de estos.

- Determinar las acciones que se van a ejecutar en correspondencia con las políticas y determinar las políticas por aplicar para cada escenario.

Si bien es evidente que, accionando sobre cualquiera de los factores determinantes, se pueden alcanzar mejoras en el estado actual de la eficiencia económica, es importante tener claro que cada provincia posee características peculiares que pueden modificar sensiblemente la factibilidad y la velocidad de respuesta de cada factor determinante. Por ello, es cardinal precisar todas las posibles acciones que se puedan desarrollar para modificar positivamente el comportamiento de los factores determinantes y así mejorar el nivel de eficiencia económica.

Una vez que se han definido y elegido las políticas para mejorar el desempeño de los factores determinantes de la eficiencia económica de la producción de frijol, será necesario verificar que se están ejecutando y que los resultados obtenidos nos aproximan al escenario apuesta para, en caso contrario, realizar las correcciones correspondientes. La idea es supervisar para asegurar, de ser necesario, el reajuste de las políticas o establecer otras nuevas. Esto es el contenido del último paso.

Paso 6: Supervisión de la mejora de la eficiencia económica de la producción de frijol en los marcos del PDIG de la provincia Santiago de Cuba.

La implementación de las políticas debe ser supervisada sistemáticamente a partir de los resultados obtenidos y su tendencia. Si estos resultados no cumplen con los parámetros establecidos, el proceso de implementación e, incluso, de diseño de políticas, debe ser revisado y reformulado en caso de ser necesario.

Esta etapa, clave en la retroalimentación del proceso de mejora de la eficiencia económica de la producción de frijol, debe basarse en el ciclo (planificar, hacer, verificar, actuar), popularizado por Deming (1989) que, mediante las funciones de planificar, hacer, verificar y actuar, dota a las entidades de las herramientas necesarias para el control y mejora de sus procesos. De acuerdo con esta filosofía, la mejora es necesaria si los resultados obtenidos no son los esperados y se precisa cambiar la forma en que se está desarrollando el proceso. En concordancia con lo anterior, la mejora estará encaminada a revisar y reformular las políticas, si es preciso, tantas veces como sea necesario, para que los resultados cumplan con los parámetros establecidos como deseables y dejen de comprometer el cumplimiento de la meta superior: el incremento de la eficiencia económica de la producción de frijol. 


\section{RESULTADOS Y DISCUSIÓN}

Una vez caracterizado el procedimiento para la evaluación prospectiva de la producción de frijol procede su aplicación en la provincia Santiago de Cuba, comenzando por el primer paso.

Paso 1: Selección del grupo de expertos.

Para la selección de los expertos se trabajó con un grupo de 11 personas. De acuerdo con los resultados obtenidos en el Coeficiente de Competencia, todos los especialistas consultados clasificaron como expertos, pues sus coeficientes son mayores o iguales que la norma generalmente aceptada de 0,8. De los 11, dos expertos son miembros del claustro de la Facultad de Agronomía de la Universidad de Oriente de Santiago de Cuba, dos de la Facultad de Ciencias Económicas y Empresariales de la Universidad de Oriente, tres de la Delegación Provincial de la Agricultura, tres vinculados directamente a la producción de granos y el Coordinador del Proyecto de Desarrollo Integral de granos de la región Oriental.

Paso 2: Elaboración de las hipótesis.

Las hipótesis para el diseño de los escenarios fueron elaboradas por los expertos teniendo en cuenta los siguientes requisitos definidos por los investigadores:

a) Deben elaborarse dos hipótesis por cada una de las tipologías de eficiencia ya definidas, es decir en total seis. (Eficiencia productiva, Eficiencia asignativa y Eficiencia asignativa)

b) Las mismas deben corresponderse con los factores que determinan dichas tipologías. La eficiencia productiva con la productividad del trabajo, el rendimiento y el costo de producción. La eficiencia asignativa con la dotación de factores y el costo de oportunidad. La eficiencia distributiva con la remuneración efectiva e ingreso neto y con la demanda efectiva y el consumo

c) Deben incluir los objetivos del PDIG en la provincia Santiago de Cuba que revela los objetivos a alcanzar en el largo plazo (2030).Implica considerar toda la proyección que contiene el Programa en cuanto a los factores determinantes por tipologías ya mencionados.

Las hipótesis elaboradas se listan a continuación:

$\mathrm{H}_{1}$ : La producción de frijol, en el año 2019, se acerca al umbral de las 14 406,77 toneladas y se mantiene un ritmo de crecimiento sostenido; en el 2030 la producción local de frijol sustituye las importaciones de este grano en la provincia Santiago de Cuba.

$\mathrm{H}_{2}$ : El incremento sostenido de la producción de frijol se realiza con un mejoramiento tendencial de los costos y del rendimiento por hectárea. Se alcanza en el 2030 una reducción del costo unitario promedio superior al $40 \%$ y un rendimiento próximo a las 1,2 toneladas por hectárea. 
$\mathrm{H}_{3}$ : Se logra incrementar el coeficiente de uso de la tierra utilizando rotaciones de cultivos, la introducción de nuevas tecnologías en el riego, la mecanización, la rehabilitación y la ampliación de infraestructuras.

$\mathrm{H}_{4}$ : La provincia mantiene su condición de líder en la producción de maíz, al tiempo que eleva la eficiencia productiva en la producción de frijol.

$\mathrm{H}_{5}$ : La expansión de la producción de frijol y el uso de nuevas tecnologías genera empleo a nivel local, eleva la remuneración de los trabajadores y mejora la relación productividad salario medio.

$\mathrm{H}_{6}$ : Mejora la oferta de frijol en los mercados locales, se eleva el consumo y se orienta la producción al tipo de frijol (colorado) de mayor demanda en el territorio.

Como se puede observar, las hipótesis se corresponden con las tipologías de eficiencia y, consecuentemente, con los factores que determinan las mismas, así como los objetivos del PDIG en la provincia Santiago de Cuba. De manera que se puede establecer una clara correspondencia entre las tipologías de eficiencia y el juego de hipótesis. Las hipótesis H1 y $\mathrm{H} 2$ están referidas a la eficiencia productiva; las H3 y H4 a la asignativa; y las H5 y H6 a la eficiencia distributiva.

Para aplicar el SMIC se escogieron, de los 11 expertos, los cinco de mayor coeficiente de competencia $(0,95)$, que lideraron tres grupos de trabajo que representaron todas las procedencias de los expertos, con un sesgo hacia aquellos que representan a los productores directos. Fueron seleccionados como líderes un experto de la Universidad de Oriente, uno de la delegación del Ministerio de la Agricultura y tres de las diferentes formas productivas vinculadas al PIDG.

Paso 3: Asignación de las probabilidades (simples y condicionales) de ocurrencia de las hipótesis.

Los resultados de la asignación de probabilidades simples de todos los expertos reconocen la hipótesis uno como la más probable, seguida de la dos con calificaciones de muy probable del orden de $60 \%$ y $40 \%$, respectivamente. Esto califica la eficiencia productiva como la de mayor probabilidad de alcanzarse, según el criterio de los expertos.

Del total de los escenarios (64 escenarios posibles), en 40 se acumula el 0,98 de probabilidad de ocurrencia de los eventos, en correspondencia con el juego de hipótesis.

A partir del análisis de los expertos se determinó considerar los primeros siete escenarios a los efectos de clasificarlos en: pesimista (64); optimista (1); apuesta (2); y alternativos $(3,5,4,9)$. Los siete escenarios acumulan el 0,70 de probabilidades de ocurrencia y están expuestos en orden descendente; teniendo el séptimo una probabilidad de ocurrencia de 0,045 .

Tabla 1. Clasificación de los escenarios probables 


\begin{tabular}{|l|l|l|l|}
\hline No. & Escenario & Probabilidad & Clasificación \\
\hline 64 & $(000000)$ & 0,215 & Pesimista \\
\hline 1 & $(111111)$ & 0,152 & Optimista \\
\hline 2 & $(111110)$ & 0,088 & Apuesta \\
\hline 3 & $(111101)$ & 0,083 & Alternativo \\
\hline 5 & $(111011)$ & 0,062 & Alternativo \\
\hline 4 & $(111100)$ & 0,055 & Alternativo \\
\hline 9 & $(110011)$ & 0,045 & Alternativo \\
\hline Probabilidad Acumulada & 0,73 & \\
\hline
\end{tabular}

La narrativa de los escenarios consideró el cumplimiento del juego de hipótesis en cada escenario y las tipologías de eficiencia.

Al asignarle el valor uno al cumplimiento de la hipótesis en cuestión y el valor cero a su no cumplimiento, y tomando en cuenta que cada tipología de eficiencia está representada con dos hipótesis, se determinó que las tipologías de eficiencia podían evaluarse de cero (mal) a dos (bien) en cada escenario. La evaluación de un punto representa el cumplimiento de solo una de las dos hipótesis y, consecuentemente, la tipología de eficiencia es evaluada de regular o de parcialmente cumplida. Esto permitió darle mayor coherencia a la narrativa de los escenarios.

Las narrativas de los escenarios, en correspondencia con los resultados obtenidos con el SMIC, fueron las siguientes:

\section{a. Escenario pesimista}

Es un escenario donde no se cumple ninguna de las hipótesis formuladas. La producción de frijol en el 2019 no se acerca al umbral de las 14400 toneladas; se deteriora el programa de sustitución de importaciones de frijol y los costos y el rendimiento tienen una tendencia que socavan los objetivos del PIDG. No se avanza en la expansión del riego y la mecanización de la producción, y el coeficiente de uso de la tierra se deteriora. La producción de maíz se reduce y la eficiencia en la producción de frijol empeora. La remuneración de los trabajadores queda por debajo del salario medio ramal y empeora la relación productividad salario medio. La oferta de frijol se reduce, elevando los precios y afectando el consumo. En este escenario todas las tipologías de eficiencia son evaluadas de mal. En la literatura especializada tal escenario suele calificarse de catastrófico. Aunque este escenario tiene la mayor probabilidad de ocurrencia como evento individual $(0,215)$, la probabilidad acumulada de los restantes eventos, donde la eficiencia económica es más favorable, es de 0,51.

b. Escenario optimista

En este escenario se cumplen todas las hipótesis y todas las tipologías de eficiencia son evaluadas de bien. La figura 1 muestra esta situación que califica como el futuro deseado. El escenario tiene como evento una probabilidad de 0,152 , inferior a la del escenario pesimista. 


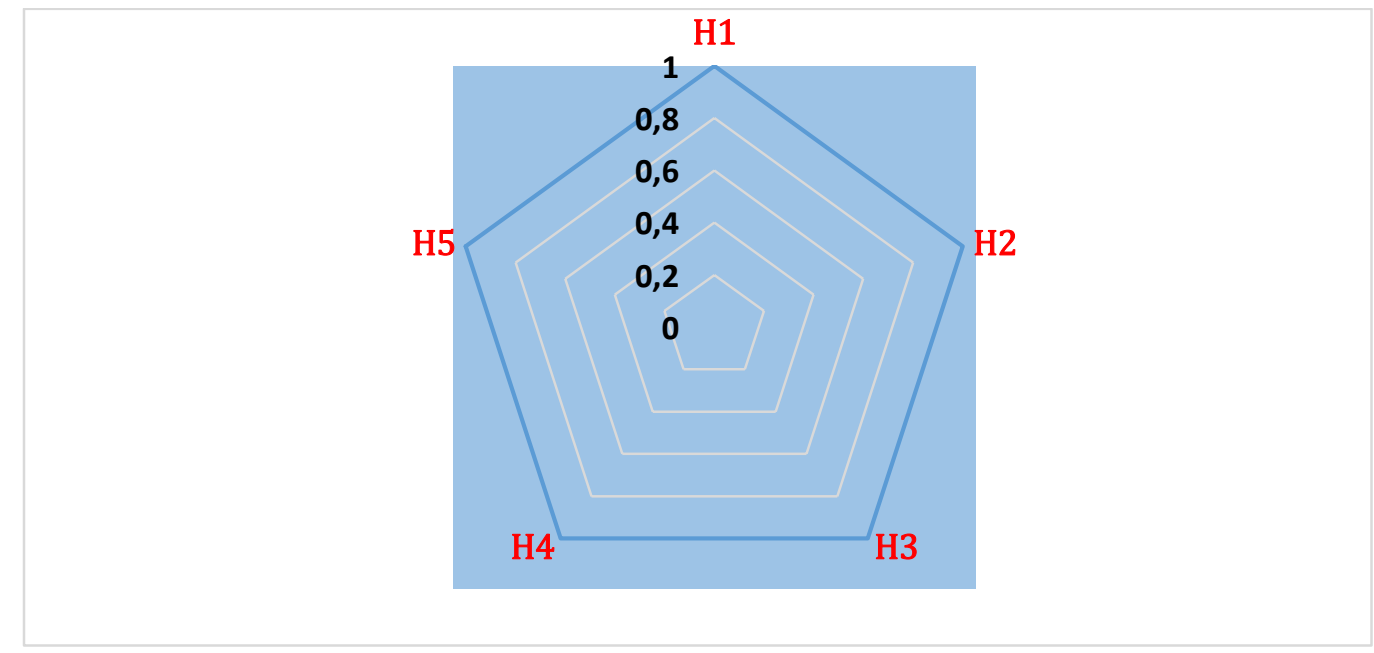

Figura 1. Escenario optimista.

\section{c. Escenario apuesta}

El escenario apuesta es seleccionado por los expertos tomando en cuenta que se cumplen las primeras cinco hipótesis. La hipótesis seis no se cumple, pero sí la cinco, que corresponde a la eficiencia distributiva. Por lo tanto, se califican de bien la eficiencia productiva y asignativa, y de regular la eficiencia distributiva.

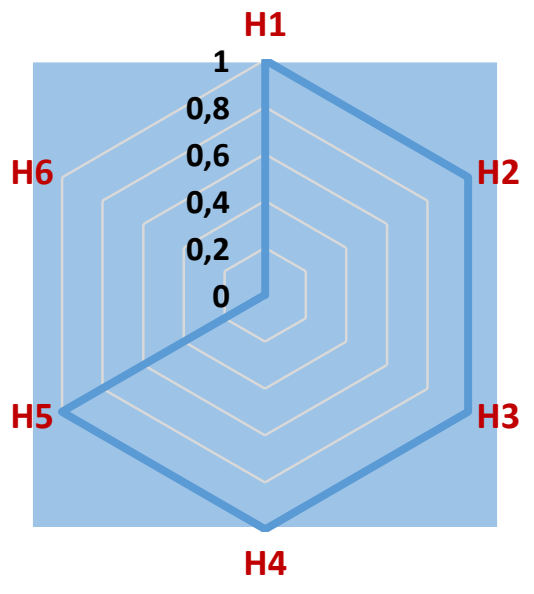

Figura 2. Escenario apuesta.

Este escenario es el de mayor probabilidad, después de los escenarios extremos (pesimista y optimista). Al mismo tiempo, debe considerarse que el escenario 3 es, desde el punto de vista de la evaluación prospectiva de la eficiencia económica, un evento similar al escenario apuesta; por consiguiente, la probabilidad de que se evalúen de bien la eficiencia productiva y la asignativa y de regular la distributiva, resulta de la suma de las 
probabilidades del escenario 3 y el escenario apuesta ${ }^{6}$; este resultado tiene una probabilidad de 0,171 , superior a la del escenario optimista.

En la narrativa del escenario apuesta debe significarse que la hipótesis seis puede incumplirse porque elevándose la oferta de frijol en los mercados locales, todavía no se cumplen los estándares de calidad que exigen los consumidores, ni se toman en cuenta suficientemente, las preferencias de estos y continúa la oferta sesgada hacia el frijol negro. Tiene lógica este escenario en el sentido de que, si bien pueden lograrse significativos avances en factores como los niveles de producción, el rendimiento, los costos, la productividad, la dotación y el empleo de factores productivos, la remuneración de la fuerza de trabajo, puede persistir cierta desconexión entre la esfera propiamente productiva y la de la distribución y consumo de frijol.

d. Escenarios alternativos.

Son el conjunto de escenario que no constituyen extremos, en cuanto al cumplimiento de las hipótesis, (que se cumplan todas las hipótesis o que no se cumpla ninguna), una vez seleccionado por los expertos el escenario apuesta. Estos escenarios juegan un importante papel en el análisis prospectivo. Son escenarios que se enfrentan al escenario apuesta como eventos probables y por tanto como alternativas que deben tomar en cuenta los decisores. El paso siguiente es analizar dentro de los escenarios alternativos, el cumplimiento de las hipótesis.

La frecuencia de cumplimiento de las hipótesis en los escenarios alternativos se presenta en la tabla 2.

Tabla 2. Frecuencia de cumplimiento de las hipótesis en los escenarios alternativos

\begin{tabular}{|c|c|c|}
\hline Hipótesis & $\begin{array}{c}\text { Frecuencia de } \\
\text { Cumplimiento/ escenarios } \\
\text { alternativos }\end{array}$ & Probabilidad acumulada $^{7}$ \\
\hline H1 & 4 & 0.245 \\
\hline H2 & 4 & 0,245 \\
\hline H3 & 3 & 0,2 \\
\hline H4 & 2 & 0,138 \\
\hline H5 & 2 & 0,107 \\
\hline H6 & 3 & 0,19 \\
\hline
\end{tabular}

La probabilidad de que se cumpla la primera hipótesis, como consecuencia de que ocurra uno de los escenarios alternativos, es del $24,5 \%$. Lo mismo sucede para la hipótesis dos. La hipótesis tres por su parte, tiene una probabilidad de que se cumpla si ocurre un escenario alternativo del $20 \%$. Esta lectura para el resto de las hipótesis permite evaluar la

\footnotetext{
${ }^{6}$ En este caso la suma de probabilidades se realiza atendiendo a la unión de un espacio muestral, tomando la siguiente fórmula: $P(A \cup B)=P(A)+P(B)-P(A \cap B)$. Como en este caso los escenarios no pueden ocurrir simultáneamente, no es posible que exista intersección entre ellos, por lo tanto, la probabilidad de que por lo menos tenga lugar uno de los dos escenarios es igual a la suma de las probabilidades de su ocurrencia individual.

${ }^{7}$ La probabilidad del cumplimiento de las hipótesis en los escenarios alternativos es igual a la suma de probabilidades de eventos disjuntos o mutuamente excluyente, y se calcula con arreglo a la fórmula expuesta en la nota al pie Nro. 6.
} 
eventualidad de ocurrencia de alguno de los escenarios alternativos frente al cumplimiento de las hipótesis. Las hipótesis uno y dos tienen la misma probabilidad de cumplirse que los escenarios alternativos de tener lugar, como un evento probable $(24,5 \%)$. Estas hipótesis responden a la eficiencia productiva, que resulta fundamental para alcanzar la eficiencia económica. La capacidad de motricidad de las mismas permitirá el cumplimiento del resto del sistema de hipótesis. Esta idea es corroborada con el análisis de sensibilidad y el coeficiente de elasticidades.

Paso 4: Análisis de sensibilidad.

En la tabla 3 se presenta el análisis de sensibilidad a través de los coeficientes de las elasticidades cruzadas de las hipótesis, que conforman los escenarios.

Obsérvese que las hipótesis uno y dos son las que mayor elasticidad de motricidad poseen, es decir, que tienen la mayor influencia sobre el resto de las hipótesis; los coeficientes calculados por el SMIC están estandarizados para una variación igual al 0,1. Lo que se interpreta como que una variación de 0,1 en H1 puede provocar una variación, por ejemplo, de 0,215 en $\mathrm{H} 2$.

La otra cuestión no menos relevante está en el hecho de que las hipótesis uno y dos son las de menor dependencia. De manera que $\mathrm{H} 1$ y H2 influyen decisivamente sobre el resto de las hipótesis, son menos dependientes y determinan la eficiencia productiva en términos de los escenarios probables de la producción de frijol en la provincia. Esto obliga a orientar las políticas para la consecución del escenario apuesta, con un importante sesgo hacia el cumplimiento de estas hipótesis en particular.

Tabla 3. Análisis de sensibilidad y coeficiente de elasticidades

\begin{tabular}{|c|c|c|c|c|c|c|c|}
\hline Hipótesis & $\mathrm{H} 1$ & $\mathrm{H} 2$ & $\mathrm{H} 3$ & $\mathrm{H} 4$ & $\mathrm{H} 5$ & $\mathrm{H} 6$ & $\begin{array}{c}\text { Elasticida } \\
\mathrm{d} \\
\text { Motricid } \\
\mathrm{ad}\end{array}$ \\
\hline $\mathrm{H} 1$ & 1 & 0,215 & 0,185 & 0,163 & 0,095 & 0,207 & 0,865 \\
\hline $\mathrm{H} 2$ & 0,161 & 1 & 0,143 & 0,143 & 0,14 & 0,186 & 0,773 \\
\hline $\mathrm{H} 3$ & 0,068 & 0,075 & 1 & 0,075 & $-0,01$ & 0,073 & 0,301 \\
\hline $\mathrm{H} 4$ & 0,033 & 0,053 & 0,054 & 1 & $-0,038$ & 0,005 & 0,184 \\
\hline $\mathrm{H} 5$ & $-0,026$ & 0,017 & $-0,038$ & $-0,05$ & 1 & 0,004 & 0,175 \\
\hline H6 & 0,013 & 0,031 & 0,001 & $-0,024$ & 0,017 & 1 & 0,085 \\
\hline $\begin{array}{c}\text { Elasticidad } \\
\text { Dependencia }\end{array}$ & 0,302 & 0,391 & 0,421 & 0,455 & 0,3 & 0,515 & \\
\hline
\end{tabular}

Paso 5: Diseño y selección de política para mejorar la eficiencia económica de la producción de frijol. 
La definición y elección de las políticas se realizó a partir de los pasos concebidos por el MULTIPOL y expuestos en el apartado del método. Se consultaron a los expertos logrando consenso sobre los distintos aspectos a evaluar. Inicialmente se definen criterios, acciones, políticas y se retoman los escenarios ya determinados en la etapa anterior. Los criterios se determinaron en términos de posibles barreras para la realización de las acciones, en los marcos de las políticas específicas en los diferentes escenarios. A continuación se listan los criterios, las acciones, las políticas y los escenarios definidos.

Tabla 4. Criterios empleados.

\begin{tabular}{|c|c|c|}
\hline Título corto & Título largo & Peso \\
\hline DI & Dependencia importadora & 1 \\
\hline DF & Dotación de factores & 1 \\
\hline RT & Requerimientos tecnológicos & 1 \\
\hline I & Incentivos & 1 \\
\hline C & Capacitación & 1 \\
\hline MA & Medio Ambiente & 1 \\
\hline
\end{tabular}


Tabla 5. Acciones propuestas.

\begin{tabular}{|c|c|c|}
\hline $\begin{array}{l}\text { Título } \\
\text { corto }\end{array}$ & Título largo & Descripción \\
\hline PLF & $\begin{array}{l}\text { Producción local de } \\
\text { frijol }\end{array}$ & Aumentar la producción local de frijol. \\
\hline $\mathrm{Pt}$ & Productividad & Elevar la productividad del trabajo. \\
\hline EFT & $\begin{array}{c}\text { Estabilidad de la fuerza } \\
\text { de trabajo }\end{array}$ & $\begin{array}{l}\text { Garantizar la estabilidad en cantidad y calidad del } \\
\text { factor trabajo. }\end{array}$ \\
\hline$A C$ & Atenciones culturales & Mejorar las atenciones culturales. \\
\hline $\mathrm{CO}$ & $\begin{array}{l}\text { Costo de oportunidad } \\
\text { (CO) }\end{array}$ & Disminuir el CO vía aumento de la eficiencia. \\
\hline I & Incentivos & $\begin{array}{l}\text { Aumentar la remuneración a los trabajadores y su } \\
\text { participación en el ingreso neto. }\end{array}$ \\
\hline CPU & $\begin{array}{c}\text { Costo de producción } \\
\text { unitario }\end{array}$ & Disminuir el costo de producción unitario. \\
\hline CUT & $\begin{array}{l}\text { Coeficiente de uso de } \\
\text { la tierra }\end{array}$ & Elevar el coeficiente de uso de la tierra. \\
\hline $\mathrm{Ca}$ & Capacitación & Elevar la capacitación. \\
\hline Rend. & Rendimiento & $\begin{array}{l}\text { Lograr un rendimiento próximo a } 1,2 \text { toneladas por } \\
\text { ha }\end{array}$ \\
\hline Co & Consumo & Aumentar el consumo de frijol. \\
\hline $\mathrm{FF}$ & $\begin{array}{c}\text { Fuentes de } \\
\text { financiamiento }\end{array}$ & $\begin{array}{l}\text { Acceder a fuentes de financiamiento para el } \\
\text { desarrollo del PDIG. }\end{array}$ \\
\hline BPA & $\begin{array}{c}\text { Buenas prácticas } \\
\text { ambientales }\end{array}$ & $\begin{array}{l}\text { Introducir buenas prácticas ambientales en la } \\
\text { producción local de frijol. }\end{array}$ \\
\hline Preferen. & Preferencias & $\begin{array}{l}\text { Orientar la producción hacia la variedad de frijol que } \\
\text { prefieren los consumidores. }\end{array}$ \\
\hline Infraest. & Infraestructura & Mejorar la infraestructura productiva. \\
\hline Semillas & Semillas & Garantizar semillas certificadas. \\
\hline Empleo & Empleo & Generar empleo a nivel local. \\
\hline
\end{tabular}


Tabla 6. Políticas diseñadas.

\begin{tabular}{|c|c|c|l|}
\hline $\begin{array}{c}\text { Título } \\
\text { corto }\end{array}$ & Título largo & Peso & Descripción \\
\hline PSI & $\begin{array}{c}\text { Política de sustitución de } \\
\text { importaciones. }\end{array}$ & 1 & Propiciar la sustitución de importaciones \\
\hline PT & Política tecnológica & 1 & $\begin{array}{l}\text { Propiciar el nivel tecnológico requerido en el } \\
\text { PDIG }\end{array}$ \\
\hline PI & Política inversionista & 1 & $\begin{array}{l}\text { Posibilitar las inversiones requeridas para } \\
\text { apoyar el PDIG }\end{array}$ \\
\hline PIN & Política de incentivos & 1 & $\begin{array}{l}\text { Propiciar la estimulación material y moral } \\
\text { de los trabajadores }\end{array}$ \\
\hline PFRH & $\begin{array}{c}\text { Política de formación de } \\
\text { R. Humanos. }\end{array}$ & 1 & Potenciar la capacitación de los trabajadores \\
\hline PA & Política ambiental & 1 & $\begin{array}{l}\text { Contribuir a la conservación del Medio } \\
\text { ambiente }\end{array}$ \\
\hline P. Social & Política Social & 1 & Potenciar el desarrollo social local. \\
\hline
\end{tabular}

Tabla 7. Escenarios construidos.

\begin{tabular}{|c|c|c|l|}
\hline Título corto & Título largo & Peso & Descripción \\
\hline EO & Escenario optimista & 1 & Se cumplen todas las hipótesis \\
\hline EA & Escenario apuesta & 1 & Se incumple la hipótesis 6 \\
\hline EA-1 & Escenario alternativo 1 & 1 & Se incumple la hipótesis 5 \\
\hline EA-2 & Escenario Alternativo 2 & 1 & Se incumple hipótesis 4 \\
\hline
\end{tabular}

Los expertos ponderaron las acciones, políticas y escenarios según los criterios seleccionados y atendiendo a la escala mencionada en la sesión del método, de forma tal que se establecieran los rankings de acciones por cada tipo de política, y los de política por cada escenario identificado.

La matriz de evaluación de las acciones en función de las políticas permitió el análisis y la clasificación de las acciones para cada política identificada y determinar un ranking por la importancia de cada una a nivel de todas las políticas y en particular en cada una de ellas. Así, por ejemplo, las acciones que más puntean son: el costo de producción unitario (17), la producción local de frijoles (15), el rendimiento (14), la productividad del trabajo (14), y las atenciones culturales (12), relacionadas directamente con la eficiencia productiva. El coeficiente de utilización de la tierra (13) y el costo de oportunidad (11) relativas a la eficiencia asignativa; y las preferencias (10) a la eficiencia distributiva. También quedan en ese primer cuadrante la estabilidad de la fuerza de trabajo y la relacionada con las fuentes de financiamiento.

Todas estas acciones constituyen una prioridad para la Política de Sustitución de Importaciones, para la Política Tecnológica, para la Inversionista y para la de Incentivos y, por tanto, conforman el núcleo priorizado de acciones en las políticas más importantes 
para el cumplimiento de las $\mathrm{H} 1$ y $\mathrm{H} 2$ que son, como resultó del análisis de sensibilidad, las que influyen decisivamente sobre el resto de las demás hipótesis. Estas políticas requieren de una atención priorizada.

A su vez, la acción más próxima a la Política Social es el empleo; y a la de Formación de Recursos Humanos se aproxima la que se refiere a la capacitación. La figura 3 muestra el plano de proximidades entre acciones y políticas.



Figura 3. Plano de proximidades entre acciones y políticas.

Otro aspecto importante a considerar es que, como se muestra en la figura 3, casi todas las acciones comentadas se sitúan en la parte superior izquierda del plano, mostrando altos valores promedio de ponderación y muy baja desviación estándar, lo que indica el nivel de coincidencia en la valoración de los expertos. Por su parte, el mapa de cercanía está indicando que la mayoría de las acciones son compartidas por las políticas identificadas. No obstante, es posible observar el alejamiento relativo, de la Política Ambiental con la acción referida a las buenas prácticas ambientales, lo que probablemente sea un reflejo de la percepción de los expertos consultados sobre el tema. Un resultado similar lo da la evaluación y la clasificación de las políticas identificadas en cada escenario (figura 4), donde todas, con excepción de la política de Formación de Recursos Humanos, son ubicadas en un primer nivel de importancia y atención. 


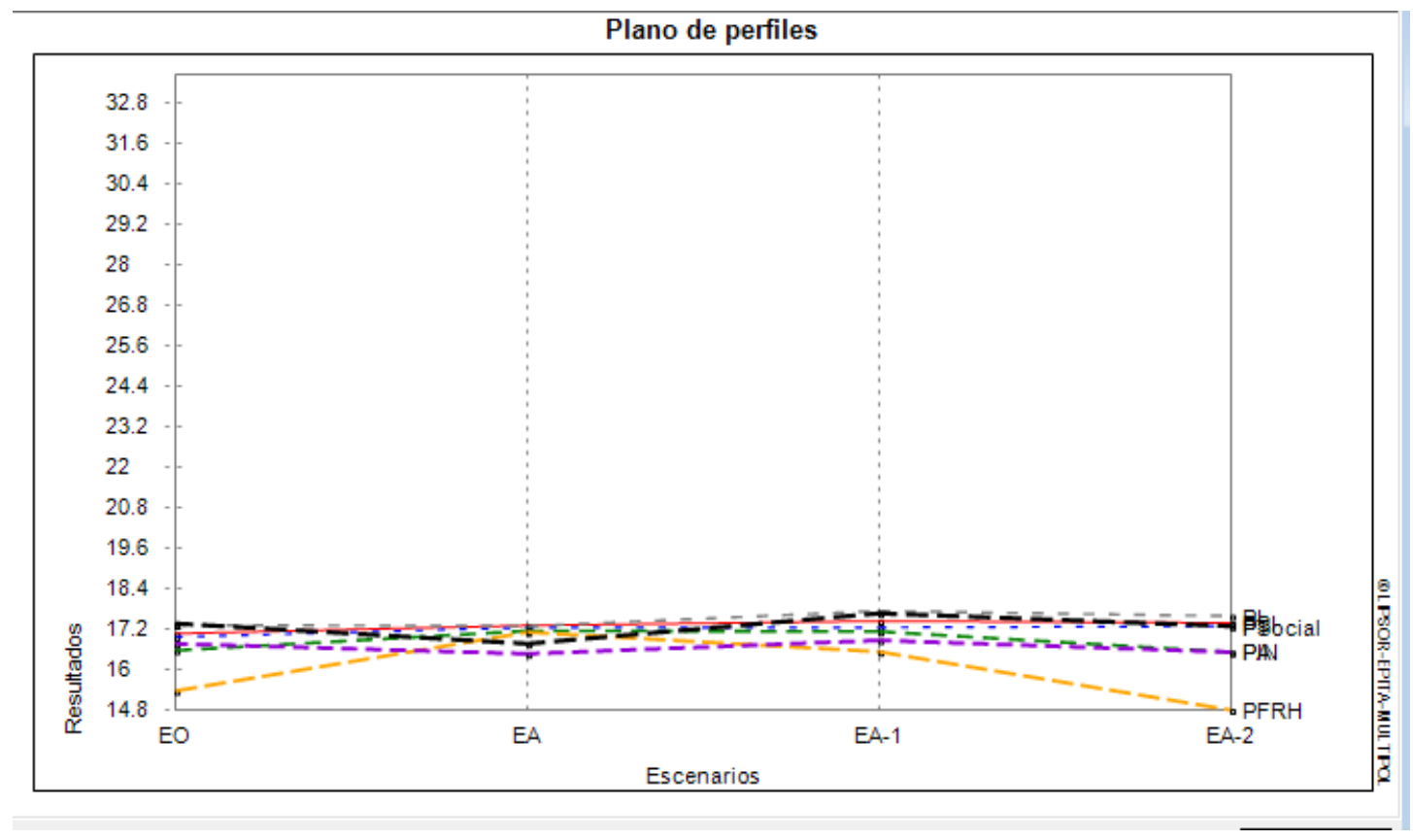

Figura 4. Plano de perfiles de escenarios.

Paso 6. Supervisión de la mejora de la eficiencia económica de la producción de frijol en la provincia Santiago de Cuba.

La supervisión del proceso de mejora de la eficiencia económica de la producción de frijol en los marcos del PDIG de la provincia Santiago de Cuba, se realiza a través de controles parciales de las políticas propuestas. En ese orden, hasta diciembre del 2016, se ha avanzado en la atención que han recibido las acciones que en esta investigación se definieron como prioritarias en las políticas trazadas, que son:

- Se ha diseñado una política de incentivos que eleve la eficiencia distributiva que, aunque tiene un mayor peso en el orden moral, se considera un resultado positivo.

- Está incluida en los objetivos del Programa la necesidad de elevar el consumo per cápita de frijol en la provincia hasta las 2,3 libras mensuales.

- Se está estudiando la posibilidad de orientar la producción hacia las preferencias de los consumidores que, como evidenció la encuesta, es la variedad de frijol rojo (colorado).

- Se han mejorado considerablemente las atenciones culturales, resultado fundamentalmente de la capacitación recibida sobre el tema.

- Los trabajadores han recibido información, han ampliado sus conocimientos y esto ha permitido sensibilizarlos con la necesidad e importancia de poner especial cuidado en las atenciones para mejorar los rendimientos.

- Se ha solicitado asistencia técnica para las empresas de los diferentes polos y se estableció un orden de prioridad con su calendario correspondiente. 
- Está ya planificado para mayo (2017) un taller sobre la dimensión ambiental del PDIG, con el objetivo de identificar los problemas ambientales que limitan la eficiencia de la producción de frijol en el territorio y la posibilidad de incorporar buenas prácticas ambientales.

\section{CONCLUSIONES}

1. La evaluación prospectiva de la eficiencia económica de la producción de frijol en la provincia Santiago de Cuba permitió:

a. El diseño de siete escenarios (pesimista, optimista, apuesta y cuatro alternativos) con el 0,70 de probabilidades de ocurrencia, a partir de la definición de seis hipótesis en correspondencia con las tipologías de eficiencia (dos hipótesis por cada tipología) y, consecuentemente, con los factores que determinan las tipologías así como con los objetivos del PDIG en la provincia Santiago de Cuba.

b. Demostrar que en el escenario apuesta como aquel de mayor probabilidad, después de los escenarios extremos (pesimista y optimista) se cumplen cinco de las seis hipótesis para un $83,3 \%$, por lo que se evalúan de bien la eficiencia productiva y la asignativa, y de regular la distributiva, pues no se cumple una de las hipótesis referidas a esta tipología de eficiencia.

c. El análisis de sensibilidad a través de los coeficientes de las elasticidades cruzadas de las hipótesis que conforman los escenarios, evidenciándose que las hipótesis uno y dos (relacionadas con la eficiencia productiva) son las que mayor elasticidad de motricidad poseen, es decir, que tienen la mayor influencia sobre el resto de las hipótesis y, a su vez, las de menor dependencia.

d. Fundamentar que la influencia de las $\mathrm{H} 1$ y las $\mathrm{H} 2$ sobre el resto de las hipótesis exige, para la consecución del escenario apuesta, priorizar las medidas de política que garanticen su cumplimiento.

e. La realización de las políticas diseñadas contribuye al logro del objetivo propuesto por el PDIG en la provincia Santiago de Cuba, al incremento de los rendimientos y la producción de granos con una reducción significativa de los costos, logrando producciones sostenibles en rubros que constituyen sustitución de importaciones (maíz y frijol) para, finalmente, coadyuvar al incremento del consumo. 


\section{BIBLIOGRAFÍA}

Bell, B. y Sarmiento, D. 2011. "Indicadores para el análisis de la eficiencia económica de empresas productoras de níquel. Caso de estudio empresa Cdte. René Ramos Latour de Nicaro". En Revista académica de economía. <http://www.eumed.net> [Consulta: 14 octubre 2015].

Cancio, D. y Barrios, C. 2010. “Evaluación de la eficiencia técnica de la producción de viandas en las empresas agropecuarias Benito Juárez y Osvaldo Herrera". En Revista académica de economía, con el Número Internacional Normalizado de publicaciones Seriadas ISSN 1696-8352. Observatorio de la Economía Latinoamericana. <htpp: //www.eumed.ne> [Consulta: 2 septiembre 2015].

Cachanosky, I. 2012. "Eficiencia técnica, eficiencia económica y eficiencia dinámica".

<htpp://www.hacer.org> [Consulta: 23 enero 2016].

Cely, A. 1999. "Metodología de los escenarios para estudios prospectivos". En Revista Ingeniería e Investigación, No. 44. <htpp: //www.bdigital.unal.edu.co> [Consulta: 3 diciembre 2015].

Cuba. Ministerio de la Agricultura [Minag]. 2015. Programa de Desarrollo Integral de Granos de la provincia Santiago de Cuba. Santiago de Cuba: autor.

Debreu, G. 1951. The Coeffcient of Resource Utilization, 19(3).

Doimeadiós, R. y Sánchez LI. 2015. "Productividad y eficiencia en la economía cubana: una aproximación empírica". En Economía y Desarrollo, vol.153 Suplemento Especial. La Habana.

Farrell, M. 1957. "The measurement of productive efficiency". Journal of the Royal Statistical Society. Series A. Vol. 120, No 3, pp. 253-290.

Färe, R., Grosskof S. y Knox L.1994. Production Frontiers. Cambridge University Press.

Ferro, G. y Romero, C. 2011. “Eficiencia y su medición en prestadores de servicios de agua potable y alcantarillado. Comisión Económica para América Latina y el Caribe" [Cepal]. <http://www.cepal.org> [Consulta: 27 mayo 2016].

Forsund, F. y Hjamarsson I. 1974. "On the Measurement of Productive Efficiency". The Swedish Journal of Economics, Vol. 76, No. 2.

García, A. 2015. “Dinamismo del sector agropecuario: condición necesaria para el desarrollo cubano". En Revista Economía y Desarrollo, Vol. 153 (Número Especial). ISSN 0252-8584. La Habana.

García, P. 2012. "Análisis de la eficiencia técnica y asignativa a través de las fronteras de costes: una aplicación a los hospitales del INSALUD. Tesis de doctorado de la Facultad de Ciencias Económicas y Empresariales". Universidad de Valladolid. 
Biblioteca virtual Miguel de Cervantes. <http: //.www.cervantesvirtual.com> [Consulta: 12 diciembre 2016].

García, A., Sánchez, D., Chadiano, M. y Núñez C. 2007. “Niveles de eficiencia de las policlínicas de Matanzas según AED”. Rev. Panamá Salud Pública, 22(2). <http: //.www.sicelosp.org> [Consulta: 28 mayo 2016].

García, R. y Sarrías, M. 2008. “La medición de la eficiencia productiva en policlínicos cubanos: un caso de estudio". En Revista especializada Economía de la Salud, 7 (3). <http: //.www.eumed.ne>

Godet, M. 1994. De la Anticipación a la Acción. Manual de Prospectiva Estratégica. Barcelona: España, Editorial Mar Combo.

Godet, M. et al. 2000. La caja de herramientas de la prospectiva estratégica. Cuarta Edición. <htpp://www.prospektier.es> [Consulta: 23 diciembre 2015].

Godet, M y Durance, P. 2011. La prospectiva estratégica para las empresas y los territorios. https://es.scribd.com. [Consulta: 2 octubre 2015].

Gómez, R. 2014. Tres ensayos sobre eficiencia económica y crecimiento regional: Capacidad empresarial, externalidades y estructura productiva. (Tesis doctoral). Departamento Economía Aplicada. Universidad Autónoma de Barcelona. Barcelona, España. FCEE. Universidad de Valladolid. <http: //.www.cervantes.virtual.com> [Consulta: 21 enero 2015].

Guerra, F. 2011. "Metodología para el análisis de la productividad en la producción de arroz. Universidad de Granma". En Revista académica de economía, con el Número Internacional Normalizado de Publicaciones Seriadas ISSN16968352.<htpp://www.eumed.net> [Consulta: 23 diciembre 2015].

Koopmans, T.1951. An analysis of production sas and efficient combination of activities. Activity analysis of production and allocation, cowles commission for research in economics. Monograph 13. New York.

Leibestein, H. 1966. "Allocative Efficiency vs X eficiency". American Economic Review, 56.

Lerner,A.1951."Teoría económica del control". .<htpp://www.catalogosuba.sisbi.uba.ar> [Consulta: 28 noviembre 2015].

Lindbeck, A. 1977. Sistemas económicos y políticas asignativas. Barcelona: España, Oikos-tan.

Martín, R. 2006. "La eficiencia en la asignación de recursos destinados a la Educación Superior. El caso de la Universidad La Laguna". ISBN: 84-7756-680-1. <http: //tesis.bbtk.ull.es> [Consulta: 2 septiembre 2015].

Medina, V. 2002. "Bases desde la Prospectiva-Previsión Territorial para el diseño del Plan Maestro del Desarrollo Integral y Sostenible del Valle del Cauca. Universidad del Valle. Quito. Ecuador". http://www.es.slideshare.net. [Consulta: 18 septiembre 2015]. 
Montes de Oca, Q. 2009. "Midiendo la eficiencia en la actividad turística mediante el análisis envolvente de datos". En TUR y DES. Vol. 2, No. 6. <http://www.eumed.net> [Consulta: 2 julio 2016].

Plank, B. 2011. “Análisis de la eficiencia en Chile”. V 72n 1 Lima, ISSN 1025-5583. <http:// www.scielo.org.pe> [Consulta: 27 mayo 2016].

Romeu, A. y Rodríguez, T. 2008. Procedimiento para la evaluación de la eficiencia técnica en la transportación de caña en las UBPC cañeras de la provincia Villa Clara. (Trabajo de Grado). <http:// www.dspace.uclv.edu.cu> [Consulta: 24 junio 2015].

Sarrías P. 2008. "Aplicación del enfoque prospectivo para la gestión del desarrollo local: caso de estudio". En Revista DELOS, Vol. 1, No. 2. http:// www.eumed.net. [Consulta: 2 junio 2015].

Sellers, R. 2005. Productividad y eficiencia en la distribución minorista en España. (Tesis doctoral. FCEE). Universidad de Alicante, España. < http: //www.tesisenred.net> [Consulta: 2 septiembre 2015].

Somoza, A. 2015. "Herramientas para la formulación de políticas: el Análisis Prospectivo en la Construcción de Escenarios Energéticos y el uso de modelos para su formulación", <https://www.nodo50.org> [Consulta: 12 noviembre 2015].

Urdaneta, F., Dios, P y Cañas J. 2013. “Estudio comparativo de la eficiencia técnica de sistemas ganaderos de doble propósito en las zonas agroeconómicas de los municipios zulianos de la cuenca del Lago de Maracaibo, Venezuela". En Revista Científica, FCV-LUZ\Vol. XXIII, No. 3. <http://www.fcv.luz.edu.ve> [Consulta: 2 junio 2016]. 